\title{
Actividad repelente de formulaciones del aceite esencial de Melaleuca quinquenervia (Cav.) S.T. Blake (Myrtales: Myrtaceae) en mosquitos
}

Maureen Leyva ${ }^{1}$, Mayda Castex¹, Domingo Montada1, Felipe Quintana², Diosanys Lezcano³ ${ }^{3}$ María del Carmen Marquetti ${ }^{1}$, Ariamys Companioni ${ }^{1}$ Jorge Anaya $^{1}$ \& Iván González ${ }^{2}$

1 Instituto de Medicina Tropical "Pedro Kouri" (IPK), Autopista Novia del Mediodía Km 6 1⁄2, Apartado 601, La Lisa, Ciudad de La Habana, Cuba.

2 Organo de Integración para la Salud, Calle N No 62, Vedado, Plaza de la Revolución, Cuba.

3 Centro Municipal de Vectores, Pinar del Rio, Cuba.

Correspondencia

Ing. M. Leyva Silva

E-mail: maureen@ipk.sld.cu

Recibido: 8 marzo 2012

Aceptado: 20 septiembre 2012

Publicado on-line: 9 octubre 2012

\section{Resumen}

En este trabajo nos trazamos como objetivo evaluar la actividad repelente de diferentes formulaciones del aceite de Melaleuca quinquinervia Cav S.T. Blake en condiciones de laboratorio y terreno. Se prepararon formulaciones etanólicas, hidroalcohólicas, hidrofílicas y en base oleosa con aceite mineral y dipropilenglicol. Se trató el antebrazo con $1 \mathrm{ml}$ de repelente y la mano fue cubierta con un guante de goma. El antebrazo fue expuesto por 3 minutos cada media hora. Se obtuvo una tendencia positiva del tiempo de protección con el aumento de la concentración. El mayor tiempo de protección observado ( 5 horas) fue con la cepa Santa Clara y la formulación de aceite al $20 \%$ en dipropilenglicol Con estos resultados se evaluó en condiciones de terreno obteniéndose tiempos de protección promedio de hora y media. El repelente formulado con dipropilenglicol y aceite de Melaleuca quinquinervia al $20 \%$ es adecuado para las labores del personal dedicado a la protección de bosques y costas.

Palabras clave: Aceite esencial, repelente, Aedes aegypti.

\begin{abstract}
Repellent activity of essential oils formulations from Melaleuca quinquenervia (Cav.) S.T. Blake (Myrtales: Myrtaceae) on mosquitoes

The study was carried out in order to evaluate the repellent activity of different formulations of Melaleuca quinquinervia Cav ST Blake oil, in laboratory and field conditions. Ethanolic, hydroalcoholic, and hydrophilic formulations were prepared in mineral oil and dipropylene glycol. In laboratory experiments the forearm was treated with $1 \mathrm{ml}$ of the repellent, and exposed against different Aedes aegypti strains by 3 minutes every half hour. The hand was protected or covered with a rubber glove. A positive protection trend was observed when the concentrations of the different formulations were increasing. The higher protection was observed (5 hours) in Aedes aegypti Santa Clara strain with $20 \%$ dipropyleneglycol oil formulations. These laboratory results were evaluated then in field conditions, obtaining average protection times of $1 \frac{1}{2}$ hours.
\end{abstract}

Key words: Essential oil, Repelent, Aedes aegypti. 


\section{Introducción}

La fiebre del dengue es una de las enfermedades más importante transmitida por mosquitos a los humanos, con más de 50 millones de casos estimados anualmente en más de 100 países (Calisher 2005, Kyle \& Harris 2008) y aproximadamente 2.5 billones de personas viven en países donde el dengue es endémico (WHO 2006). El crecimiento poblacional mundial asociado a la urbanización no planificada, en especial en países donde predominan hacinamiento y deterioro en el suministro de agua, así como el aumento de residuos sólidos que favorecen el hábitat de las larvas, contribuyen al incremento de este padecimiento. Es preocupante que la distribución geográfica y la severidad de los brotes del dengue aumentan convirtiendo las poblaciones humanas en vulnerables (Kyle \& Harris 2008, Franco et al. 2010, Guzmán 2010).

El control químico es la principal medida recurrente, en caso de epidemias de dengue, para garantizar una disminución drástica de las infestaciones del vector. Ante el aumento de las enfermedades emergentes y remergentes y el alto costo de las campañas de control antivectoriales, el mundo encamina sus esfuerzos en la búsqueda de alternativas más ecológicas, menos contaminantes y dañinas al ser humano.

Las propiedades de las plantas han sido utilizadas desde la antigüedad con fines medicinales, insecticidas y para repeler, aunque fuese momentáneamente, la picadura de insectos, sirviendo algunas como precursoras de los insecticidas que hoy conocemos en la actualidad (Ware \& Whitacre 2004).

Por definición, los repelentes son sustancias que actúan de forma local evitando que un insecto en pleno vuelo se pose y pique en la piel (Blackwell et al. 2003, Choochate et al. 2007). Hoy en día, se comercializa repelentes sintéticos y naturales o mezcla de ellos. El más conocido es el DEET (N-N- dietil-m-toluamida). Es el más eficaz y persistente en la piel (Fradin \& Day 2002, Isman et al. 2006). Debido a su patrón de uso limitado, la exposición al DEET proviene sólo de aplicaciones personales. No se utiliza en la agricultura y, por lo tanto, no se encuentra presente en los alimentos. Se descompone lentamente en el suelo y con bastante rapidez en la atmósfera (NAT 2000).

Algunos repelentes basados en plantas son comparables con los sintéticos aunque tiendan a ser de menor duración. Entre las plantas productoras de aceites esenciales, con actividad repelente, se han estudiado las pertenecientes a géneros, como Cymbopogon spp., Eucalyptus spp. y Ocimum spp. Estas plantas han sido tradicionalmente utilizadas para repeler a los mosquitos en zonas selváticas como el Amazonas boliviano (Moore et al. 2007). Estos géneros producen la mayoría de los repelentes naturales utilizados en el mundo (Trongtokit et al. 2005)

Melaleuca quinquenervia es una planta comúnmente conocida como Cayeput (Oviedo 2011). Constituye una especie invasora y no deseada, que crece en el humedal de importancia internacional de la Ciénaga de Zapata, en la provincia de Matanzas, Cuba, causando pérdidas económicas y a la biodiversidad botánica. Esta planta ha sido evaluada, comprobándose un efecto antiprotozoario (Rodríguez-Pérez et al. 2006) antimalárico (Fernández-Calienes et al. 2008), antihelmíntico (Roig 1974), bactericida y fungicida (GuevaraPérez 2010), además de larvicida y repelente (Leyva et al. 2008, Amer \& Mehlhorn 2006), respectivamente.

Ante la posibilidad de ampliar los usos de esta planta como un recurso ecosostenible, este trabajo tiene como objetivo evaluar la actividad repelente de diferentes formulaciones del aceite esencial de Melaleuca quinquinervia en condiciones de laboratorio y de terreno.

\section{Material y métodos}

\section{Colecta del material y obtención del aceite}

Para la obtención del aceite esencial, primeramente se recolectaron las partes aéreas de $M$. quinquenervia (ejemplar registrado con el número 42678 en el Instituto de Ecología y Sistemática, Cuba)

El aceite fue extraído por el método de destilación por arrastre con vapor de la parte aérea de las plantas utilizando un equipo Clevenguer. El destilador tiene forma cilíndrica y un volumen de 421 , fabricado con láminas de $3 \mathrm{~mm}$ de espesor. En la parte superior, presenta una abertura con cierre hermético para introducir el material a destilar, así como una tubuladura para la salida de los vapores. Aproximadamente a $1 / 3$ de su altura tiene insertado, en su interior, una rejilla metálica con orificios de $3 \mathrm{~mm}$ de diámetro para soportar el material vegetal y permitir el paso del vapor a través de la misma. Esta rejilla no está fijada, para permitir su 
cambio de posición de forma tal que el material pueda ser embebido en agua. La fuente de calor utilizada fue gas combustible. El condensador está construido de tubos de acero inoxidable con $1 \mathrm{~mm}$ de espesor, es del tipo serpentín y está sumergido en una cuba con capacidad para 175 l. Éste tiene entrada y salida para el agua de enfriamiento. El área de enfriamiento es de $0,16 \mathrm{~m}^{2}$. El separador de aceite esencial o vaso florentino fabricado es del tipo para aceites esenciales menos densos que el agua. Tiene forma cilíndrica con un cuello más estrecho, donde se coloca un embudo metálico con vástago acodado lo suficientemente largo $(1 / 3$ a $1 / 4$ del fondo) por donde es descargado el condensado.

\section{Identificación de los componentes}

El análisis cromatográfico fue realizado por parte del Laboratorio de Productos Naturales del Centro de Nacional de Investigaciones Científicas (CN IC). Para la identificación de los componentes, se empleó un cromatógrafo de gases $6890 \mathrm{~N}$ acoplado a detector selectivo de masas 5975 Binert (Agilent, EUA), con una columna HP-5 Ms (30 $\mathrm{mm} \times 0,25 \mathrm{~mm}$ d.i. y $0,25 \mu \mathrm{m}$ de espesor de película). La programación de temperaturas fue: de $60{ }^{\circ} \mathrm{C}(1 \mathrm{~min}$ isotérmico $)$ hasta $240{ }^{\circ} \mathrm{C}$ a $3{ }^{\circ} \mathrm{C} / \mathrm{min}$, con 15 min a la temperatura final. El flujo del gas portador (He) fue de $1 \mathrm{ml} / \mathrm{min}$. El inyector, en modo split $(1: 10)$ se calentó a $250^{\circ} \mathrm{C}$. Las temperaturas de la interfaz, fuente de ionización y cuadrupolo fueron: $250{ }^{\circ} \mathrm{C}, 230^{\circ} \mathrm{C}$ y $150{ }^{\circ} \mathrm{C}$, respectivamente. La energía de ionización fue $70 \mathrm{eV}$ y la adquisición se realizó desde 20 a $600 \mathrm{~m} / \mathrm{z}$. El volumen de inyección fue $0,5 \mu \mathrm{l}$. La identificación se llevó a cabo por comparación de los espectros obtenidos con los de las bibliotecas NIST 1998 y Willey 275, y por comparación de los índices de Kovats con los reportados en la literatura.

\section{Bioensayos de laboratorio}

Se utilizó, primeramente, una cepa de Ae. aegypti (Linneo, 1762) de referencia, susceptible a insecticidas, nombrada Rockefeller y una cepa del terreno nombrada Santa Clara, proveniente de la provincia con el mismo nombre, obtenida en fase larval y estabilizada en el insectario. Para los bioensayos se utilizaron cajas de $30 \times 30 \times 30 \mathrm{~cm}$, con 200 hembras de 3 a 6 días de edad sin alimentar. Se evaluó el aceite puro (AEPURO) y luego se prepararon cremas con el aceite esencial (CM10, CBIO, CF10, CF2 y CF3) y una solución hidroal- cohólica (AH20+ET), además de formulaciones al $8,10,15$ y $20 \%$ en base oleosa con aceite mineral (M8A, M10A, M15A y M20A) y M8D, M10D, M15D y M20D en dipropilenglicol. Los protocolos de elaboración de cremas y formulaciones no pueden incluirse debido a que están siendo patentados.

Para ambos bioensayos, se trató el antebrazo con $1 \mathrm{ml}$ de repelente y la mano fue cubierta con un guante de goma. Se utilizó un control positivo $(\mathrm{C}+)$ con DEET al $20 \%$ y un control negativo (C-) al que se le aplicó el solvente utilizado en cada formulación, cuando ésta era evaluada. El antebrazo fue expuesto 3 minutos cada 30 minutos (WHO 2009). Las picaduras de cada mano de los voluntarios se cuantificaron y el porcentaje de protección (PP) se calculó de acuerdo con la fórmula:

$$
P P=\frac{N c-N t}{N c} \times 100
$$

donde $\mathrm{Nc}=$ número de picaduras en la mano control en determinado período de tiempo, $\mathrm{Nt}=$ número de picaduras en la mano tratada en el mismo período (Rueda et al. 1998, Frances \& Wirtz 2005).

Se tomó en cuenta el criterio de tiempo de protección al primer tiempo después de aplicado el tratamiento, donde se observa una picada seguida de otra confirmatoria en el mismo periodo o en el periodo de exposición siguiente.

\section{Bioensayos de terreno}

Las pruebas de campo se realizaron en 12 lugares diferentes que abarcaban la Ciénaga de Zapata, el municipio Martí, Matanzas y el sur de la Provincia La Habana, Cuba. Para esos estudios se utilizaron 30 voluntarios del personal dedicado a la protección de bosques y costas. Se realizaron entre los meses de agosto y septiembre, típicos de verano, que se caracterizan por tener las temperaturas de $25-32^{\circ} \mathrm{C}$ en la zona occidental y las densidades más altas de mosquitos costeros como $O$ chlerotatus taeniorhynchus (Wiedemann, 1821).

En cada lugar se instruyó, previamente, al personal sobre la metodología a emplear y se entregó un modelo para anotar lugar de la prueba, fecha, persona que realizó la prueba, hora de aplicación, tiempo de protección, tipo de insectos prevalentes, claves de la concentración del aceite. Se evaluaron las formulaciones al 10, 15 y $20 \%$ con base en dipropilenglicol. La aplicación fue realizada en 
los horarios de la noche y madrugada. Se tomó en cuenta el criterio de tiempo de protección al primer tiempo después del tratamiento, donde se observa una picada seguida de otra confirmatoria en el mismo o en el periodo de exposición siguiente.

\section{Resultados}

\section{Identificación de los componentes}

Mediante el análisis cromatográfico del aceite esencial de $M$. quinquinervia se determinaron 37 compuestos, de los cuales se identificaron 35 . El monoterpenoide 1,8-cineol fue el componente mayoritario seguido por el sesquiterpenoide hidroxilado viridiflorol, por los monoterpenoides limoneno, $\alpha$-pineno, $\beta$-pineno y $\alpha$-terpineol, y por los sesquiterpenoides $\alpha$-terpenilacetato, $\beta$-cariofileno, óxido de cariofileno y globulol, todos los cuales presentaron contenidos superiores al $1 \%$ (Morales-Rico et al. 2012).

\section{Bioensayos de laboratorio}

En el estudio realizado ni el aceite esencial puro de $M$. quinquinervia, ni las cremas hidrofílicas, ni la solución etanólica, ofrecieron la protección esperada con la cepa Rockefeller. Con el aceite puro se obtuvieron más de 5 picaduras a los 33 minutos de la aplicación y con las formulaciones en base de crema las picaduras oscilaron desde 6 hasta más de 20 picaduras, a los 3 minutos de aplicadas, excepto una donde se observaron más de 20 picaduras a los 33 minutos. Por no haber tenido resultados satisfactorios con estas formulaciones, no se tabularon ni graficaron.

De las formulaciones a base de aceite mineral, el porcentaje de protección más bajo observado en la cepa Rockefeller se obtuvo con la formulación al $10 \%$ (M10A), casi comparable con el control negativo. En el resto de las formulaciones con aceite mineral el porcentaje de protección fue mayor, aunque fueron perdiendo sucesivamente su actividad en el tiempo. De las formulaciones con

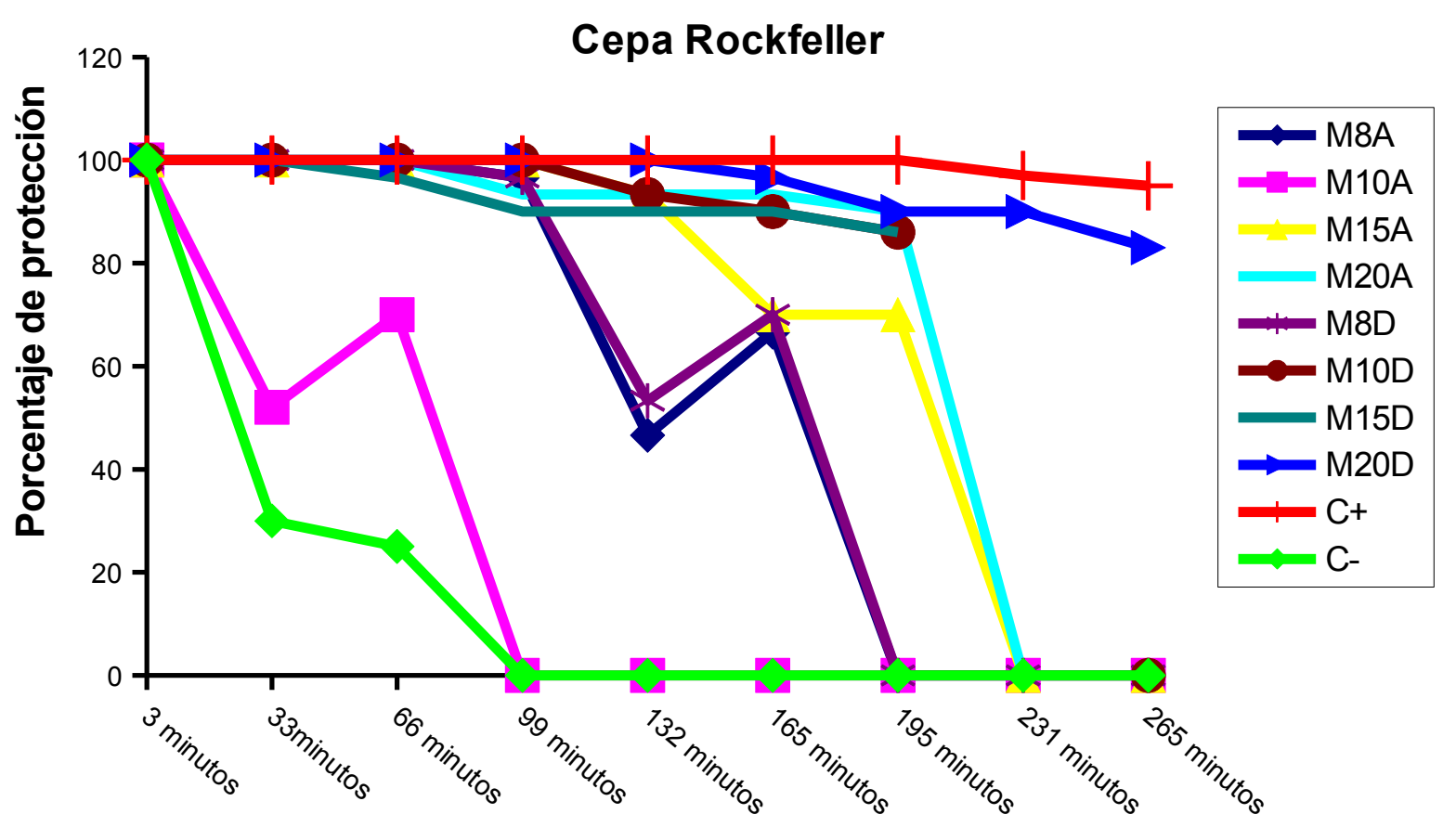

Tiempos de protección

Figura 1. Porcentaje de protección que brindaron las diferentes formulaciones en la cepa de Aedes aegypti Rockefeller. Formulaciones al 8 , 10, 15 y $20 \%$ en base oleosa con aceite mineral (M8A, M10A, M15A y M20A) y dipropilenglicol (M8D, M10D, M15D y M20D). Control positivo $(\mathrm{C}+$ ) con DEET, al $20 \%$, y control negativo (C-), con el solvente utilizado en cada formulación.

Figure 1. Percentage of protection times provided by different formulations on Rockefeller Aedes aegypti strain. $8,10,15$ and $20 \%$ formulations in mineral oil (M8A, M10A, M15A y M20A) and dipropylene glycol (M8D, M10D, M15D and M20D). Positive control (C+) with $20 \%$ DEET and negative control (C-) with the used solvent for each formulation. 


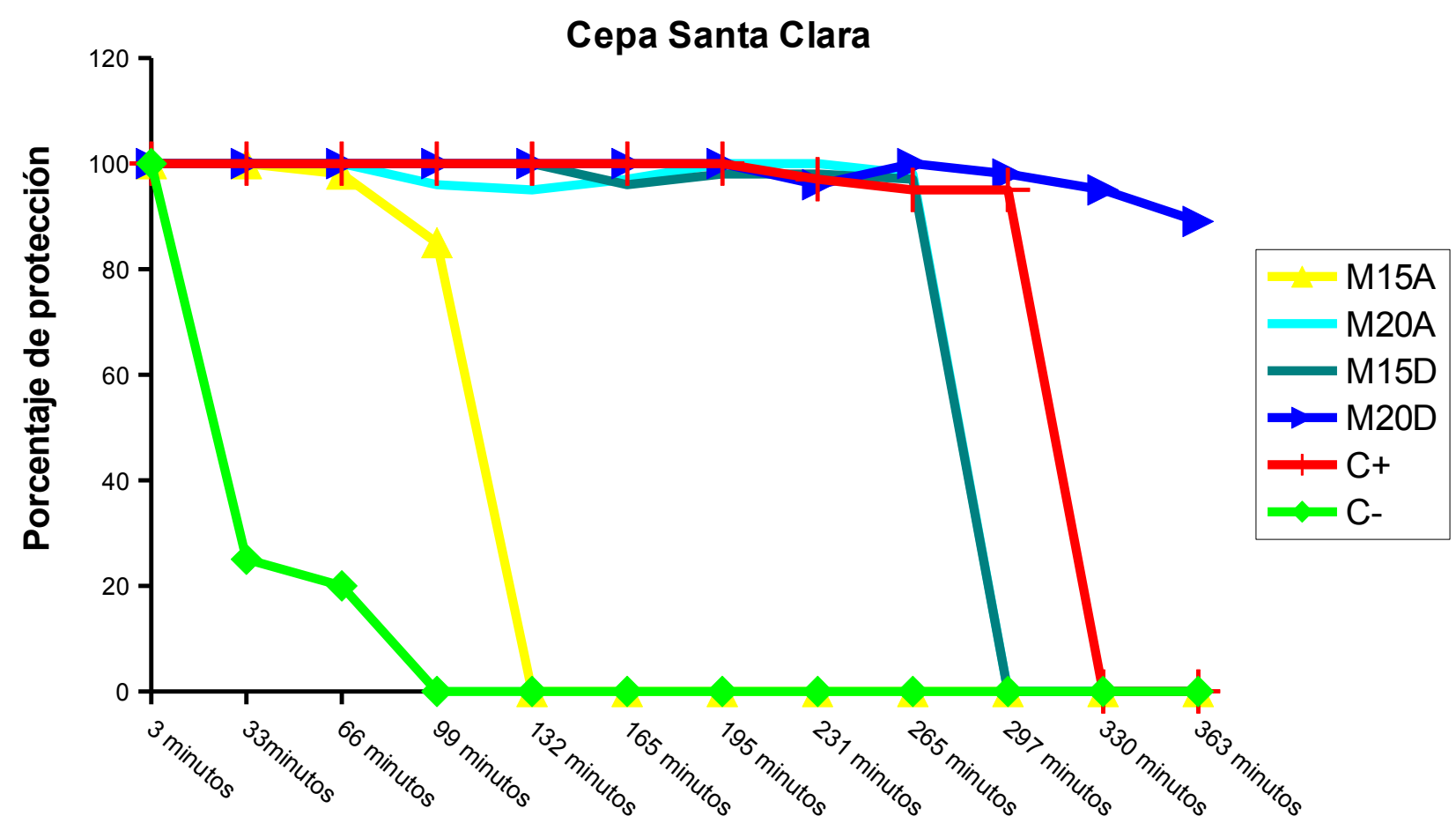

Tiempos de protección

Figura 2. Porcentaje de protección que brindaron las diferentes formulaciones en la cepa de Aedes aegypti Santa Clara. Formulaciones al $15 \mathrm{y}$ $20 \%$ en base oleosa con aceite mineral (M15A y M20A) y dipropilenglicol (M15D y M20D). Control positivo (C+) con DEET, al $20 \%$, y control negativo (C-), con el solvente utilizado en cada formulación.

Figure 2. Percentage of protection times provided by different formulations on Santa Clara Aedes aegypti strain. 15 and $20 \%$ formulations in mineral oil (M15A and M20A) and dipropylene glycol (M15D and M20D). Positive control (C+) with $20 \%$ DEET and negative control (C-) with the used solvent for each formulation.

dipropilenglicol, las que brindaron mayor efecto protector fueron M15D y M20D (Fig. 1).

$\mathrm{Al}$ evaluarse las formulaciones con la cepa Santa Clara, los mejores resultados se encontraron en las formulaciones con dipropilenglicol. La preparación M20D mostró el mayor tiempo casi igual o mayor que el control positivo con DEET con un mayor porcentaje de protección, cuando se compara con el control positivo (Fig.2).

En general, los tiempos de protección de las formulaciones a base de aceite mineral y dipropilenglicol son bajos si comparamos con el control positivo, teniendo en cuenta que los bioensayos de laboratorio se realizaron en condiciones ideales de temperatura y humedad. Sin embargo, la formulación con aceite de $M$. quiquinervia al $20 \%$ y dipropilenglicol presenta el mejor tiempo (Tabla 1). $\mathrm{Al}$ realizar el análisis estadístico de los resultados, mediante un ANOVA bifactorial, los mismos mostraron diferencias ligeramente significativas, cuando analizamos el efecto combinado de concentración y solvente en los tiempos de protección medios observados en la cepa Rockefeller. Hay diferencia entre los solventes, independiente de la concentración, a favor del dipropilenglicol y existe una tendencia positiva del tiempo de protección con el aumento de la concentración. Cuando se compararon los resultados en ambas cepas Rockefeller y Santa Clara, con las concentraciones al $15 \%$ y $20 \%$ del aceite esencial, el análisis mostró una diferencia ligeramente significativa ( $p=$ $0.049^{*}$ ) en efecto combinado de concentración y solvente. De forma general, en este análisis el solvente dipropilenglicol mostró mejores resultados que el aceite mineral. Con la cepa de terreno Santa Clara se obtuvo el mayor tiempo de protección a la concentración del $20 \%$ que con la cepa de laboratorio Rockefeller.

\section{Bioensayos de terreno}

En la figura 3, se muestra el tiempo promedio de protección al evaluar las concentraciones de 10, 15 y $20 \%$, (M10D, M15D y M20D), respectivamente. El mayor tiempo de protección se muestra 


\begin{tabular}{|c|l|c|c|}
\hline \multirow{2}{*}{ Concentración } & Solvente & $\begin{array}{c}\text { TPTM } \\
\text { cepa } \\
\text { Rockfeller }\end{array}$ & $\begin{array}{c}\text { TPTM } \\
\text { cepa } \\
\text { Santa Clara }\end{array}$ \\
\hline \multirow{2}{*}{$8 \%$} & DPG & $123.7(2.6 \mathrm{~h})$ & - \\
\cline { 2 - 4 } & $\begin{array}{l}\text { Aceite } \\
\text { Mineral }\end{array}$ & $132(2.2 \mathrm{~h})$ & - \\
\hline \multirow{2}{*}{$10 \%$} & DPG & $180.7(3.0 \mathrm{~h})$ & - \\
\cline { 2 - 4 } & $\begin{array}{l}\text { Aceite } \\
\text { Mineral }\end{array}$ & $49.5(0.76 \mathrm{~h})$ & - \\
\hline \multirow{2}{*}{$15 \%$} & DPG & $140.2(2.3 \mathrm{~h})$ & $206.2(3.4 \mathrm{~h})$ \\
\cline { 2 - 4 } & $\begin{array}{l}\text { Aceite } \\
\text { Mineral }\end{array}$ & $156.7(2.6 \mathrm{~h})$ & $82.5(1.3 \mathrm{~h})$ \\
\hline \multirow{2}{*}{$20 \%$} & DPG & $187.5(3.1 \mathrm{~h})$ & $\mathbf{3 0 5 . 2} \mathbf{( 5 . 8} \mathbf{h})$ \\
\cline { 2 - 4 } & $\begin{array}{l}\text { Aceite } \\
\text { Mineral }\end{array}$ & $140.2(2.3 \mathrm{~h})$ & $206.2(3.4 \mathrm{~h})$ \\
\hline Control + & DEET $20 \%$ & $253.5(4.22 \mathrm{~h})$ & $255(4.5 \mathrm{~h})$ \\
\hline Control - & c/ solvente & 0 & 0 \\
\hline
\end{tabular}

Tabla 1. Tiempos de protección total en minutos (TPTM) obtenidos para cada una de las formulaciones de aceite esencial de Melaleuca quinquinervia (Cayeput) evaluadas ante la cepa de Aedes aegypti Rockefeller y Santa Clara en condiciones de laboratorio.

Table 1. Total protection times in minutes (TPTM) for each formulations of essential oil of Melaleuca quinquinervia (Cajeput) obtained with Rockefeller and Santa Clara Aedes aegypti strain under laboratory conditions.

con la formulación al $20 \%$ corroborando los resultados de laboratorio. Al realizar el análisis estadístico mediante una prueba de Kruskal-Wallis los mismos no muestran diferencia significativa para una $p=0.05$, aunque el tiempo de mayor protección continúa siendo para la formulación al $20 \%$ en dipropilenglicol. Como un efecto colateral se reportaron repelencia o muerte de las hormigas (Wasmania auropunctata (Roger, 1863)) y efectos curativos en la piel de algunos combatientes que presentaban lesiones producidas por hongos $\mathrm{u}$ otras infecciones, al aplicar sucesivamente el repelente. Los gastos de repelente por persona en una noche, a la intemperie, oscilaron entre 8 y 10 gramos. En ningún voluntario se observó toxicidad ni irritabilidad dérmica u oftálmica, lo cual se había estudiado anteriormente (García 2010a, b).

\section{Discusión}

Algunos de los metabolitos secundarios presentes en los aceites esenciales suelen ser producidos por la planta, con el fin de disuadir el ataque de insectos (Gatehouse 2002) y pueden variar su composición considerablemente entre las especies de plantas y dentro de la misma variedad en diferentes áreas geográficas (Zygadlo \& Juliani 2003).

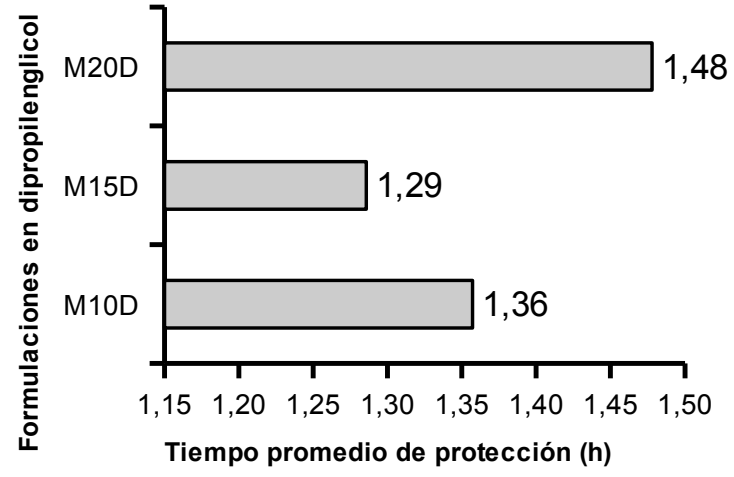

Figura 3. Tiempo de protección medio (h) de las formulaciones al $10,15,20 \%$ del aceite de Melaleuca quinquinervia en dipropilenglicol probadas en el terreno.

Figure 3. Times protection average (h) of different formulations of Melaleuca quinquinervia $(10,15,20 \%)$ oil in dipropylene glycol tested in field conditions.

Un gran número de aceites esenciales provenientes de diferentes familias han demostrado repelencia contra especies de artrópodos (Nerio et al. 2010). Estudios realizados por diversos autores plantean que algunos monoterpenos, como el $\alpha$ terpineol, $\beta$-mirceno, linolool, 1.8-cineol, 4-terpineol, timol, carvacrol, $\alpha$-pineno y $\beta$-pineno, limoneno, alcanfor poseen algún tipo actividad insecticida (Araujo et al. 2003, Carvalho et al. 2003, Panella et al 2005, Byeoung-Soo et al 2005, Lucia et al. 2007, Gillij et al. 2008) y entre los sesquiterpenos el $\beta$-cariofileno es citado como un repelente fuerte en contra de Ae. aegypti (Jaenson et al. 2006, Trongtokit et al. 2005). Aunque las propiedades repelentes de aceites esenciales varían regularmente entre plantas, al parecer, la bioactividad está asociada con la presencia conjunta de monoterpenos y sesquiterpenos en la composición del aceite (Sukumar et al. 1991, Jaenson et al. 2006, Kiran \& Devi 2007).

En el estudio realizado al aceite de $M$. quinquinervia, los monoterpenos 1.8-cineol y limoneno, además del sesquiterpeno viridiflorol, representan más del $60 \%$ de los componentes mayoritarios (Morales-Rico et al. 2012), por lo que somos del criterio que la actividad conjunta de estos metabolitos es la que brinda la repelencia al aceite, por ser reportados anteriormente por otros autores como responsables de algún tipo de actividad insecticida.

Por lo general, la mezcla de todos los componentes de un aceite hace que posean mayor actividad biológica, en comparación con los componentes aislados (Gillij et al. 2008). Inclusive, se ha comprobado que la mezcla de varios aceites tie- 
nen mejor resultado que los aceites por separados, debido a que aumenta la concentración de los componentes (Liu et al. 2006). En nuestra experiencia, comprobamos esto con la actividad larvicida de un aceite de trementina, obtenido de la destilación de resina de pinos, los cuales en su composición reportaban $\alpha$ y $\beta$-pineno. Al comprobar el $\alpha$-pineno aislado, obtuvimos la CL50 más alta que la CL50 del aceite de trementina propiamente, por lo que se corroboró que ambos monoterpenos producían un efecto sinérgico letal (Leyva et al. 2012).

En estudios realizados con cámaras de excitorepelencia, con el aceite esencial de $M$. leucadendra, en Ae. aegypti, el mismo mostró fuerte actividad irritante y repelente por el tiempo tan corto de permanencia y el rápido escape de las cámaras (Noosidum et al. 2008). Dentro de los muchos estudios realizados para demostrar y explicar la repelencia, está el estudio realizados con 20 terpenoides con dos grupos funcionales, uno con carga negativa, que contenía esteres o hidroxilos de etanol, y uno con carga positiva, que contenía grupos alcanos. Se encontró que los que poseen la carga positiva, en el extremo de la cadena hidrogenocarbonada, son los más propicios para las interacciones con los quimiorreceptores (Wang et al., 2008). El modelo desarrollado mostró que el momento dipolar y el punto de ebullición están estrechamente relacionados con la actividad repelente. El primero podría estar relacionada con las interacciones electrostáticas específicas con el receptor, mientras que el punto de ebullición a una determinada presión de vapor puede determinar la duración del tiempo de contacto con los quimiosensilios olfativos de los mosquitos (Wang et al. 2008).

El aumento de la actividad repelente también depende en gran medida de la composición del producto. En nuestro caso, el aceite de M. quinquinervia probado puro tuvo poca acción repelente. Resultados similares fueron obtenidos por Gi1lij et al. (2008) al evaluar 14 aceites al $90 \%$ de pureza, donde encontró 2 con muy poca acción repelente debido a que solo protegieron por 3 y 6 minutos. Este efecto puede deberse a que los aceites esenciales actúan comúnmente en una fase de vapor (Zhu et al. 2001), lo que los hace eficaces durante un periodo de tiempo relativamente corto (Barnard 2000). Este hecho está probablemente relacionado con su alta volatilidad, lo que es mejorado a través del desarrollo de formulaciones para mantener los ingredientes activos sobre la piel durante períodos de tiempo más largos.

En estudios realizados con formulaciones de Cymbopogon citratos (DC.) Stapf., la eficacia de las diferentes formulaciones fue mayor en una base hidrofílica seguido de la base emulsionable y en base oleosa (Oyedele et al. 2002). Sin embargo, en nuestro estudio cuando se prepararon diferentes cremas con el aceite esencial tampoco se obtuvieron buenos resultados, es decir la crema no ofreció diferencia con respecto al aceite puro.

En diferentes formulaciones farmacéuticas, solidas o semisólidas para acción tópica, el dipropilenglicol es utilizado por sus características aditivas e inocuas, por su excelente solubilidad en agua, aceites e hidrocarburos, junto con su escaso olor, bajo potencial de irritación de la piel, baja toxicidad, distribución consistente de isómeros y excelente calidad. Es capaz de crear una monocapa en la epidermis que permite la persistencia y la biodisponibilidad de los ingredientes activos. Este factor hace que, en nuestro caso, los componentes mayoritarios del aceite perduren mayor tiempo y su acción se sinergice. El aceite mineral, por su excesiva solubilidad, no tiene esta capacidad que facilita la permanencia del aceite esencial durante más tiempo.

Este factor debe tenerse en cuenta para las mejoras de las formulaciones, debido a que el aroma natural de la planta hace agradable el olor del producto y que el hombre pase desapercibido en zonas naturales donde es aplicado. Existen estudios donde se han añadidos algunos materiales fijadores para hacer perdurar el efecto de repelencia, como la parafina, ácido salicilírico y vainillina (Tawatsin et al. 2001, Oyedele et al. 2002, Blackwell et al. 2003). Este último, no sólo aumenta significativamente los tiempos de protección, sino que también lo hace efectivo para diferentes especies de mosquitos (Tawatsin et al. 2001, Choochate et al. 2007)

En el estudio realizado en terreno con las formulaciones al 10, 15, $20 \%$ en dipropilenglicol, se encontraron tiempos más bajos que los obtenidos en condiciones de laboratorio. Este resultado era esperado debido a que las poblaciones de insectos hematófagos en las costas en los meses de verano son altas. Aun así, entre los voluntarios hubo aceptación debido al olor natural y el hecho de no ofrecer inconvenientes por volverlo a aplicar cuando terminaba el tiempo de protección. $\mathrm{Mu}$ chos observaron acción terapéutica en los lugares donde había afectaciones dermatológicas debido a 
picadas de insectos. Somos del criterio de que, si las formulaciones se mejoran con algunos aditivos podemos aumentar su perdurabilidad en la piel y tener a nuestra disposición una alternativa más para la protección contra la picada de mosquitos y otros insectos.

El uso de las plantas como repelentes naturales es prometedor, siempre que encaminemos los esfuerzos a la mejor opción: proteger el medio ambiente y encontrar soluciones para el control de las enfermedades de forma saludable y sostenible. En algunos lugares se trabaja con el 3.8-diolparamentano (PMD) debido a que es el único repelente a base de plantas que se ha recomendado para su uso en zonas endémicas de Malaria por el CDC (Centro para el Control de Enfermedades), debido a su eficacia clínica demostrada para prevenir la malaria (Zielinski-Gutierrez et al. 2010). El mismo se obtiene de los residuos del destilado de Corymbia citriodora (Hook.) K.D. Hill \& L.A.S. Johnson (Myrtaceae) (Barasa et al. 2002), pero también puede ser obtenido de otras plantas, el cual proporciona una protección de varias horas (Carroll \& Laye. 2006), siendo su efecto muy similar al DEET, con un futuro prometedor en el campo de la repelencia. Por ahora, debemos encaminar nuestros estudios y esfuerzos con esta fuente ecosostenible de M. quinquinervia.

En este trabajo, se obtuvo una tendencia positiva del tiempo de protección con el aumento de la concentración. El mayor tiempo de protección observado en el estudio de laboratorio fue en la formulación de aceite de M. quinquinervia al $20 \%$ en dipropilenglicol frente a la cepa Santa Clara. El repelente formulado a base de dipropilenglicol y aceite esencial de M. quinquinervia al $20 \%$ mostró igualmente buenos resultados en el terreno y es adecuado para las labores cotidianas del personal dedicado a la protección de bosques y costas.

\section{Agradecimientos}

A todos los voluntarios que participaron en el bioensayo de terreno. Al profesor Juan Enrique Tacoronte $\mathrm{PhD}$ por su valiosa revisión y contribución a este documento.

\section{Referencias}

Amer A \& Mehlhorn H. 2006. Repellency effect of fortyone essential oils against Aedes, Anopheles, and Culex mosquitoes. Parasitolgy Research 99 (4):
478-490.

Araujo ECC, Silveira ER, Lima MAS, Neto MA, Andrade IL \& Lima MAA. 2003. Insecticidal activity and chemical composition of volatile oils from Hyptis martisii Benth. Journal of Agricultural and Food Chemistry 51 (13): 3760-3762.

Barasa SS, Ndiege IO, Lwande W \& Hassanali A. 2002. Repellent activities of stereoisomers of $p$-menthane3,8-diols against Anopheles gambiae (Diptera: Culicidae). Journal Medical Entomology 39: 736-741.

Barnard D. 2000. Repellents and toxicants for personal protection. En Pesticide Evaluation Scheme (WHO PES). WHO/CDS/WHOPES/GCDPP/2000.5. Geneva Switzerland.

Blackwell A, Stuart E \& Estambale BA. 2003. The repellent and antifeedant activity of oil of Myrica gale oil against Aedes aegypti mosquitoes and its enhancement by the addition of salicyluric acid. The Journal of the Royal College of Physicians of Edinburgh 33 (3): 209-214.

Byeoung-Soo P, Won-Sik C, Jeong-Han K, Kap-Ho K, Spng-Eun L. 2005. Monoterpenes from Thyme (Thymus vulgaris) as potential mosquito repellents. Journal American Mosquito Control Association 21 (1): 80-83.

Carroll SP \& Loye J. 2006. PMD, a registered botanical mosquito repellent with deet-like efficacy. Journal American Mosquito Control Association 22 (3): 507514.

Calisher CH. 2005. Persistent emergence of dengue. Emergent Infectolgy Disease 11: 738-739.

Carvalho AF, Melo VM, Carveiro AA, Machoo MI, Beantim MB \& Rabelo EF. 2003.Larvicidal activity of the essential oil from Lippia sidoide against Aedes aegypti. Memorias do Instituto Oswaldo Cruz 98 (4): 569-571.

Choochote W, Chaithong U, Kamsuk K, Jitpakdi A, Tippawangkosol $P$, Tuetun $B$, Champakaew D \& Pitasawat B. 2007. Repellent activity of selected essential oils against Aedes aegypti. Fitoterapia 78 (5): 359-364.

Fernández-Calienes A, Mendiola J, Scull R, Vermeersch M, Cos P \& Maes L. 2008. In vitro anti-microbial activity of the Cuban medicinal plants Simarouba glauca DC, Melaleuca leucadendron $L$ and Artemisia absinthium L. Memorias do Instituto Oswaldo Cruz 103 (6): 615-618.

Fradin MS \& Day JF. 2002.Comparative efficacy of insect repellents against mosquito bites. New England Journal of Medicine 347 (1): 13-18.

Franco C, Hynes NA, Bouri N \& Henderson DA.2010. The dengue threat to the United States. Biosecurity and Bioterrorism: Biodefense Strategy, Practice, and Science 8 (3): 273-276.

Frances SP \& Wirtz RA. 2005. Repellents: past, present, and future. Journal of American Mosquito Control Association 21 (sp1): 1-3.

Gillij YG, Gleiser RM \& Zygadlo JA. 2008. Mosquito repellent activity of essential oils of aromatic plants growing in Argentina. Bioresource Technology 99 (7): 2507-2515.

García G. 2010a. Ensayo de irritabilidad dérmica del aceite de M. leucadendron L. proveniente del OIPS. 
CEIEB. IFAL. P 10/53. La Habana Cuba.

García G. 2010b. Ensayo sobre toxicidad oral del aceite de M. leucadendron proveniente del OIPS. CEIEB. IFAL. P 10/56. La Habana Cuba.

Gatehouse JA. 2002. Plant resistance towards insect herbivores: a dynamic interaction. New Phytologist 156 (2): 145-159.

Guevara-Pérez E, Cabrera-Dorta T, Peña-Ruiz T, Fernández-Rodríguez CJ, Quintana-Guevara I \& Fernández- Rodríguez E. 2010. Efecto antimicrobiano de hojas de Melaleuca leucadendron ( $L$ ) que crece en la Ciénaga de Zapata. Revista Médica Electrónica 32 (4). Disponible en URL: http://www. revmatanzas.sld.cu/revista $\% 20$ medica/ano\%202010/vol4\%20 2010/tema04.htm.

Guzmán MG. 2010. Dengue: a continuing global threat Nature Reviews Microbiology 8: S7-S16.

Isman MB. 2006. Botanical insecticides, deterrents, and repellents in modern agriculture and an increasingly regulated world. Annual Review of Entomology 51: 45-66.

Jaenson TG, Palsson K \& Borg-Karlson AK. 2006. Evaluation of extracts and oils of mosquito (Diptera: Culicidae) repellent plants from Sweden and GuineaBissau. Journal Medical of Entomology 43 (1): 113119.

Kiran S \& Devi P. 2007. Evaluation of mosquitocidal activity of essential oil and sesquiterpenes from leaves of Chloroxylon swietenia DC. Parasitology Research 101: 413-418.

Kyle JL. \& Harris E.2008. Global spread and persistence of dengue. Annual Review of Microbiology 62: 7192.

Leyva M, Tacoronte JE, Marquetti MC, Tiomno O, Montada D \& Mesa A. 2008. Actividad insecticida de aceites esenciales de plantas en larvas de Aedes aegypti (Diptera: Culicidae). Revista Cubana de Medicina Tropical 60 (1): 78-82.

Leyva M, Tiomno O,Tacoronte JE, Marquetti MC, Montada D. 2012. Essential Plant Oils and Insecticidal Activity in Culex quinquefasciatus. En Insecticides Pest Engineering (Perveen F., ed.) Ed. Intech. 221238 pp. Disponible en http://www.intechopen.com/ books/insecticides-pest-engineering.

Liu CH, Mishra AK, Tan RX, Tang C, Yang HY \& Shen F. 2006. Repellent and insecticidal activities of essential oils from Artemisia princeps and Cinnamomum camphora and their effect on seed germination of wheat and broad bean. Bioresource Technology 97(15:) 1969-1973.

Lucia A, Gonzalez Audino P, Saccacini E, Licastro S, Zerba E\& Masuh H. 2007.Larvicidal effect of Eucalyptus grandis essential oil and turpentine and their major components on Ae aegypti larvae. Journal of the American Mosquito Control Association 23(3): 293-303.

Moore SJ, Hill N, Ruiz C \& Cameron MM. 2007. Field evaluation of traditionally used plant-based insect repellents and fumigants against the malaria vector Anopheles darlingi in Riberalta, Bolivian Amazon. Journal of Medical Entomology. 44 (4): 624-630.

Morales-Rico C, Marrero-Delange D, González-Canava-
cioloV, Quintana-Ramos F \& González- Camejo I. (En prensa). Caracterización físico-química del aceite esencial de las partes aéreas de Melaleuca quinquenervia. Comunicación corta: Revista CENIC. Ciencias Químicas. 2012.

Nerio LS, Olivero-Verbel J \& Stashenko E. 2010. Repellent activity of essential oils: A review. Bioresource Technology 101(1): 372-378.

Noosidum A, Prabaripai A, Chareonviriyaphap T \& Chandrapatya A. 2008. Excito-repellency properties of essential oils from Melaleuca leucadendron L, Litsea cubeba (Lour.) Persoon, and Litsea salicifolia (Nees) on Aedes aegypti mosquitoes. Journal of Vector Ecology 33 (2): 305-312.

NAT. 2000. National Pesticides Telecommunications Network DEET (General Fact Sheet). Oregon State University. Disponible en http://npic.orst.edu/factsheets/DEETgen.pdf

Oviedo R. 2011. "CERTIFICO". Melaleuca quinquenervia (Cav). Instituto de Ecología y Sistemática. Herbario Nacional, Onaney Muñiz. La Habana Cuba.

Oyedele A, Gbolade O, Sosan A, Adewoyin M, Soyelu F \& Orafidiya O. 2002. Formulation of an effective mosquito-repellent topical product from Lemongrass oil. Phytomedicine 9: 259-262.

Panella NA, Dolan MC, Karchesy JJ, Xiong Y \& PeraltaCruz J. 2005. Use of novel compounds for pest control: insecticidal and acaricidal activity of essential oils components from heartwood of Alaska yellow cedar. Journal of Medical Entomology 42 (3): 35258.

Rodríguez-Pérez M, Martínez JM, Rivero LR, Álvarez $\mathrm{HMH}$, Valdez AF, Rodríguez DA, et al. 2006. Evaluación de la actividad antimalárica de algunas plantas utilizadas en la medicina tradicional cubana. Revista Ciencias Farmaceutica Básica Aplicada 27 (3): 197205. Disponible en http://www.win2pdf.com.

Rueda LM, Rutledge LC \& Gupta RK. 1998. Effect of skin abrasions on the efficacy of the repellent deet against Aedes aegypti. Journal of the American Mosquito Control Association 14: 178-182.

Sukumar K, Perich M \& Boobar L. 1991. Botanical derivatives in mosquito control: a review. Journal of the American Mosquito Control Association 7: 210-237.

Tawatsin A, Wratten SD, Scott RR, Thavara U \& Techandamrongsin Y. 2001. Repellency of volatile oils from plants against three mosquito vectors. Journal of Vector Ecology 26 (1): 76-82.

Trongtokit $Y$, Rongsriyam Y, Komalamisra N \& Apiwathnasorn C. 2005. Comparative repellency of 38 essential oils against mosquito bites. Phytotherapy Research. 19 (4): 303-309.

Wang Z, Song J, Chen J, Song Z, Shang S, Jiang Z \& Han Z. 2008. Study of mosquito repellents from terpenoid with a six-member-ring. Bioorganical \& Medical Chemistry Letters 18 (9): 2854-2859.

Ware GW \& Whitacre DM. 2004. The Pesticide Book. Willoughby, Ohio: Meister Media Worldwide.

WHO. 2006. Report of the Scientific Working Group on meeting on Dengue. TDR/ SWG/08. Geneva Switzerland.

WHO. 2009. Pesticide Evaluation Scheme (WHOPES): 
Guidelines for efficacy testing of mosquito repellents for human skin. WHO/HTM/NTD/ WHOPES/ 2009.4. Geneva Switzerland.

Zielinski-Gutierrez E, Raw R, Nasci S. \& Brogdon W.G. 2010. Protection against mosquitoes, ticks and other insects and arthropods. En CDC Health Information for International Travel (V) (Brunette G.W., ed.) Atlanta: Centers for Disease Control and Prevention, disponible en http://wwwnc.cdc.gov/travel/page/yellowbook-2012-home.htm (accedido el 5 de marzo de
2012).

Zygadlo JA \& Juliani HR. 2004. Recent progress in medicinal plants. En Phytochemistry and Pharmacology (Majundar DK, Govil JN \& Singh VK, eds.). Texas: Research Periodicals \& Books Publishing House, pp. 273-281.

Zhu BC, Henderson G, Chen F, Fei H \& Laine RA. 2001. Evaluation of vetiver oil and seven insect-active essential oils against the Formosan subterranean termite. Journal of Chemical Ecology 27(8): 1617-1625. 